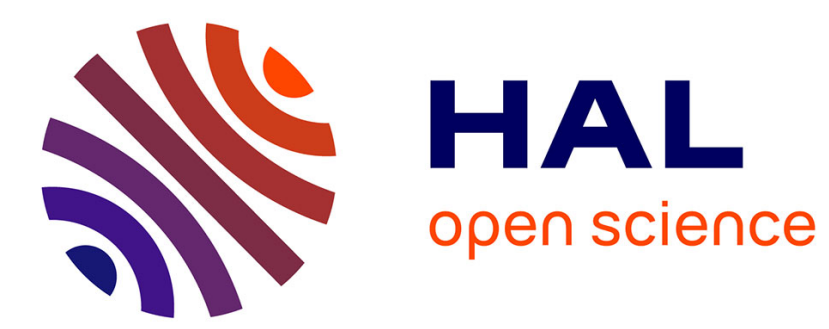

\title{
EXTRACTING THE TRANSIENT FROM PERCUSSIVE SOUNDS
}

\author{
J. Meillier, J. Laroche
}

\section{To cite this version:}

J. Meillier, J. Laroche. EXTRACTING THE TRANSIENT FROM PERCUSSIVE SOUNDS. Journal de Physique IV Proceedings, 1992, 02 (C1), pp.C1-89-C1-92. 10.1051/jp4:1992115 . jpa-00251108

\section{HAL Id: jpa-00251108 https://hal.science/jpa-00251108}

Submitted on 1 Jan 1992

HAL is a multi-disciplinary open access archive for the deposit and dissemination of scientific research documents, whether they are published or not. The documents may come from teaching and research institutions in France or abroad, or from public or private research centers.
L'archive ouverte pluridisciplinaire HAL, est destinée au dépôt et à la diffusion de documents scientifiques de niveau recherche, publiés ou non, émanant des établissements d'enseignement et de recherche français ou étrangers, des laboratoires publics ou privés. 


\title{
EXTRACTING THE TRANSIENT FROM PERCUSSIVE SOUNDS
}

\author{
J.L. MEILLIER et J. LAROCHE* \\ Laboratoire d'Acoustique de l'Université du Maine, Route de Lavah, BP. 535, F-72017 Le Mans \\ cedex, France \\ *Télécom Paris, 46 nue Barraul, F-75634 Paris cedex 13, France
}

\begin{abstract}
This work is concerned with the modeling of percussive acoustical signals, and focuses mostly on the sound's non-stationary onset. The original signal is modeled by a resonating filter fed with a short excitation-signal. The resonating filter reflects the acoustical properties of the instrument's body (frequencies and damping factors of the vibration modes), and the excitation signal models the way the instrument was set into vibration. The resonating filter is calculated by use of a cumulated time-frequency representation of the original signal. The excitation signal is obtained by use of inverse filtering with an optional regularization procedure. Analysis examples are given and some applications are proposed and discussed.
\end{abstract}

\section{A Introduction}

This work is concerned primarily with the analysis of the sound of percussive instruments. Three major preoccupations are on our mind: first, we are interested in obtaining high quality syntheses. More precisely, we will focus on the first hundred milliseconds of the signal which classical synthesis methods have great difficulty rendering properly. Second, we want to extract the signal's "non-sinusoidal" contents found in the first hundred milliseconds. The idea here is to be able to further analyze and possibly model this "non-sinusoidal" signal. Finally, this work can be seen as an attempt to make a connection between physical modeling, which deals with the physics of music instruments, and signal analysis, which deals with the sounds the instruments produce: our excitation signal can be compared with the data obtained by the physical modeling of the interaction between the exciter and the resonator. In this paper, we will represent the original signal by a classical source/filter model in which the filter condenses the sinusoidal nature of the signal's resonance, and the source corresponds to the signal's first hundred non-stationary milliseconds. The choice of the model is presented and justified in the first part. The calculation of the filter and of the source signal is derived in the second part. In the third part we present some examples that illustrate our method, and point to future investigations.

\section{B The Signal Model}

As suggested before, the observed signal $s_{n}$ is supposed to be the output of a resonating filter $H(z)$ fed with an excitation signal $e_{n}$. In order to separate the influence of the exciter and the response of the instrument we decided that filter $H(z)$ should be related only to the instrument's body, regardless of the excitation:

The resonating part of the output signal can usually be modeled by a sum of decaying sinusoids [8] whose frequencies and damping factors are independent of the excitation, at least in the range of linear motion. Their relative amplitudes and phases on the contrary depend heavily on the way the instrument was physically excited. Similarly, filter $H(z)$ should be related only to the values of frequencies and of damping factors. The distribution of amplitudes and phases is characterized by the exciting signal. Our choice is as follows:

Filter $H(z)$ is a resonating filter composed of a sum of first order cells with unit amplitude and zero phase.

$$
H(z)=\sum_{i=1}^{L} \frac{1}{1-z_{i} z^{-1}}+\frac{1}{1-\bar{z}_{i} z^{-1}}
$$


Its impulse response is a sum of decaying cosine waves with equal amplitudes, zero phases, frequencies $f_{i}$ and damping factors $\alpha_{i}$. The values of frequencies and damping factors are given by

$$
f_{i}=\frac{S R}{2 \pi} \operatorname{Arg}\left(z_{i}\right) \quad \text { and } \quad \alpha_{i}=-S R \log \left(\left|z_{i}\right|\right)
$$

where frequencies are expressed in $\mathrm{Hz}$, damping factors in $\mathrm{s}^{-1}$. SR denotes the sampling rate, $\operatorname{Arg}(z)$ is the argument of complex number $z,|z|$ its modulus and $\bar{z}$ its complex conjugate.

An equivalent expression for $H(z)$ is the classical rational expression:

$$
H(z)=\frac{z^{-2 L+1} B(z)}{z^{-2 L} A(z)} \quad \text { with } \quad A(z)=\prod_{i=1}^{L}\left[\left(z-z_{i}\right)\left(z-\bar{z}_{i}\right)\right] \quad \text { and } \quad B(z)=\frac{d A(z)}{d z}
$$

This choice of filter $H(z)$ is somewhat arbitrary: the first order cells might have been attributed different weightings and relative phases. Our choice will be justified later on. The determination of parameters $L$ and $z_{i}$ is discussed in the following part.

According to our model, the excitation signal $e_{n}$ represents the interaction between the exciter and the body of the instrument and therefore should be of short duration. It contains the information about the relative amplitudes and phases of the various sinusoidal components, along with the supplementary information that cannot be modeled by decaying sinusoids.

We will now turn to the problems of determining filter $H(z)$ and calculating the excitation signal $e_{n}$.

\section{Determining the filter and calculating the excitation}

\section{Estimating filter $H(z)$}

As we saw, the complex numbers $z_{i}$ are related to the frequencies $f_{i}$ and damping factors $\alpha_{i}$ of the sinusoids by eq. (2). We need only estimate the values of the frequencies and damping factors to completely characterize our filter. Several methods may be used to perform this task.

Parametric methods immediately come to mind: Prony's method [1], the MUSIC [2] or ESPRIT [3] methods yield direct estimates of frequencies and damping factors. Unfortunately, they can be used only on short-duration signals (less than about 600 samples). For longer durations, they require that the signal be undersampled or decomposed into several sub-bands. In that case, the amount of computation makes parametric methods less attractive.

Another alternative is the use of classical Fourier Transform. This is the most efficient method with regard to computational cost, even though it involves the calculation of several spectra. Its successive steps will now be outlined.

The method we use is inspired by Schroeder's method for the estimation of the impulse response of concert halls [4], and its time-frequency generalization ("Energy-Decay Relief") [5]. In the first step, successive power-spectra $P_{i}(f)$ are calculated on a window located at increasing times $n_{i}$. These spectra are then cumulated, starting from the last one, finishing by the first one. This procedure yields a $3-\mathrm{D}$ power spectrum $P_{\text {cumulated }}(f, i)$ :

$$
P_{\text {cumulated }}(f, i)=\sum_{j \geq i}\left|P_{j}(f)\right|^{2} \quad \text { with } \quad P_{i}(f)=\sum_{k=1}^{N} s_{k+n_{i}} \cdot w_{k} . e x p^{j .2 x . k . J / N}
$$

where $w_{k}$ represents a weighting window, usually a Hamming window. $P_{\text {cumulated }}(f, i)$ is the value at frequency $f$ of the spectra cumulated over the range $j \geq i$. Fig. 1 presents a $3-D$ plot of $P_{\text {eumulated }}(f, i)$.

In the second step, a peak detection is performed on the cumulated power-spectrum $P_{\text {cumulated }}(f, 1)$ to detect the sinusoidal components in the signal and calculate their frequency. The precise value of frequencies are obtained by use of a standard second-degree interpolation. Finally, the damping factors are estimated from the slope of the 3-D power spectrum, when $f$ is kept constant and $i$ is permitted to vary. The cumulation does not affect the values of damping factors, and has a smoothing effect that facilitates their estimation.

Once the poles $z_{i}$ have been computed, the filter $H(z)$ is derived from formula (3). At that point, a stability problem can occur. If the original sound is harmonic with a low fundamental, the coefficients of filters $A(z)$ and $B(z)$ can exhibit extremely large values, of the order of magnitude of the binomial coefficients: $a_{i} \sim L ! /(i !(L-i) !)$ where $L$ is the degree of polynomial $A(z)$. To avoid the resulting computation-instability problem, polynomial $A(z)$ can be factored into several smaller-degree polynomials $A(z)=A_{1}(z) \cdot A_{2}(z) \ldots . . A_{k}(z)$. As can be seen in Fig. 1 , the filter's magnitude transfer function exhibits peaks of nearly equal amplitude. Once the filter has been determined, the excitation signal can be calculated.

\section{Calculating the excitation signal}

The excitation signal can be calculated by use of inverse filtering methods. In our case, the filter $H(z)$ given by eq. (1) can be shown to be minimum-phase provided $\left|z_{i}\right|<1$. In other words, if the sinusoids in the original signal 

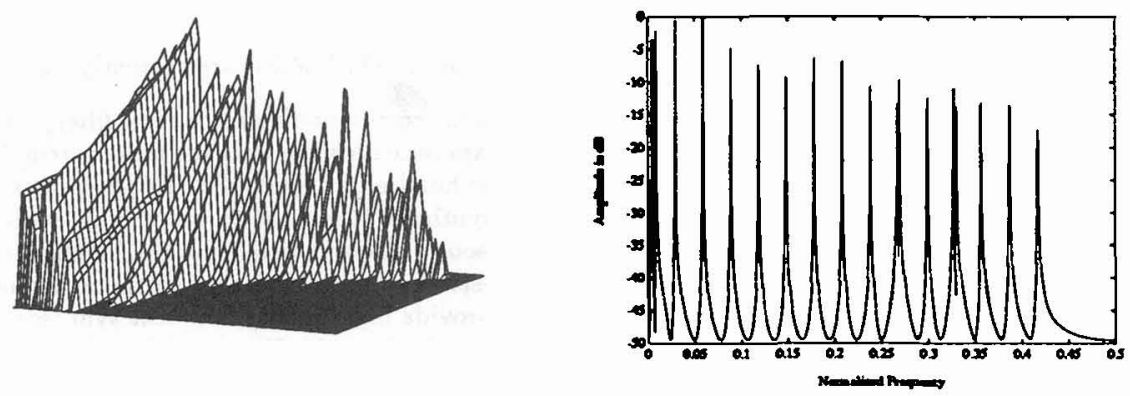

Figure 1: Left: three-dimensional plot of the Energy-Decay Relief of a guitar sound. Right: Magnitude transfer function of the corresponding filter $H(z)$

are indeed damped, filter $H(z)$ has both its poles and its zeros inside the unit circle. Consequently, the inverse filter is stable. However, this condition is not sufficient to ensure that inverse filtering is feasible [6].

Indeed, direct inverse filtering often leads to unreliable results because of the ill-conditioned nature of the problem: if the magnitude transfer function of filter $H(z)$ exhibits deep valleys, the solution of the inverse filtering problem can be extremely unstable with respect to the output signal. This problem can be circumvented in two ways:

- One can use a regularization technique. Rather than the exact solution of the inverse filtering problem, we choose an approximate solution which also minimizes a regularity criterion. A possible criterion could be that the excitation signal have little energy in the areas where the filter's magnitude transfer function is near-zero. A fast implementation exists, which makes use of fast fourier transform to perform the calculation of the regularized solution. See [6] for further information about regularization techniques.

- Another solution consists in using a filter $H(z)$ for which the inverse filtering problem is not ill-conditioned. This remark motivates our choice of filter $H(z)$. Defined as in eq. (1), the filter's impulse response is a sum of damped cosine waves with zero phase: because of the impulse response's strong discontinuity at $n=0$, the magnitude transfer function never approaches zero. The corresponding inverse filtering problem turns out to be quite stable.

Other choices of filter $H(z)$ could have been made: instead of a sum of cosine waves, we might have preferred a sinusoidal impulse response leading to the following expression: $G(z)=\sum_{j=1}^{L} 1 / i\left[\left(1-z_{j} z^{-1}\right)^{-1}-(1-\right.$ $\left.\bar{z}_{j} z^{-1}\right)^{-1}$. In yet another possibility, the first order cells could have been laid in a serial implementation rather than in our parallel implementation. The transfer function would have been: $F(z)=\prod_{j=1}^{L}\left(1-z_{j} z^{-1}\right)^{-1}(1-$ $\left.\bar{z} ; z^{-1}\right)^{-1}$. Such filters can be shown to be minimum-phase too, but their magnitude transfer functions exhibit near-zero values between the resonances: a regularization technique has to be used.

With our choice of filter $H(z)$, the excitation signal can be obtained by direct inverse filtering, which amounts to simple filtering by: $H^{-1}(z)=A(z) / B(z)$. Fig. 2 presents the excitation signals corresponding to two notes played on the same guitar. The two signals appear to be of short duration.
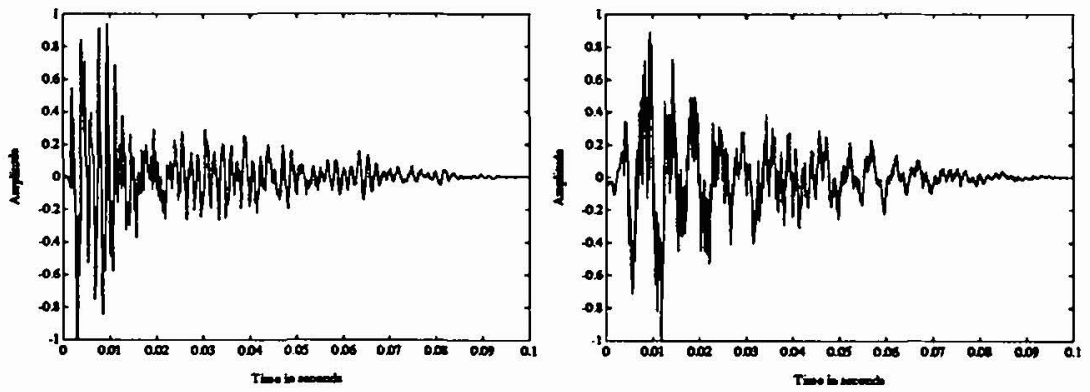

Figure 2: Left: excitation signal of a guitar, note E3. Right: excitation signal of the same instrument, note B3 


\section{Then, what do we do with it?}

The source/filter model can be used in a number of applications, of which a few are currently being investigated:

1. Synthesis and cross-synthesis: if the excitation is fed into the corresponding resonating filter, the original sound is obtained, up to the small regularization error. Our experiments show that for most percussion sounds, the excitation signal is of high amplitude during the first few hundred milliseconds, then decreases rapidly toward zero. Even when a truncated excitation is used, the synthesis turns out to be of high quality: in contrast with standard additive-synthesis [1], the onset of the sound is quite well rendered by this technique. Note that very efficient source/filter methods exist to synthesize harmonic or nearly harmonic signals [7]. Used in conjunction with our excitation signal, these methods provide high-quality, low-cost syntheses.

The excitation corresponding to an instrument can also be fed into the filter modeling another instrument, in a process often called "cross-synthesis" [8]. Interesting sound effects can be obtained that way.

2. One major advantage of our technique is the availability of the excitation signal $e_{n}$. Ideally, the excitation signals corresponding to different notes played in an identical manner on the same instrument should exhibit some similarities. This remains to be verified to validate our model.

3. From a physical modeling point of view, the interaction between the exciter and the resonator is usually a non-linear phenomenon. A number of analysis techniques for non-linear identification could be applied to our excitation signals, in order to obtain a model of the excitation itself: in particular, techniques using higher-order statistics.

4. Finally, considering the fact that the sound's onset is of the highest importance in the human identification of sources, we might try to classify and identify acoustical sounds on the basis of their respective excitation signals.

\section{References}

[1] J. Laroche, "Etude d'un Système d'Analyse et de Synthèse Utilisant la Méthode de Prony. Application aux Instruments de Musique de Type Percussif," Doctoral Dissertation, ENST, Paris, October 1989.

[2] R. O. Smith, "Multiple Emitter Location and Signal Parameter Estimation," Proc. RADC Spectrum Estimation Workshop (Rome, NY) pp. 243-258, 1979.

[3] Y. Hua, T. K. Sarkar, "Matrix Pencil Method for Estimating Parameters of Exponentially Damped/Undamped Sinusoids in Noise," IEEE Trans. Acoust., Speech, Signal Processing, vol. ASSP-38 (No. 5), pp. 814-824, May 1990.

[4] M.R. Schroeder "New method for mesuring reverberation time," Journal of the Acoustical Society of America, vol. 37 , pp. 232-235, 1965.

[5] J.M. Jot "An Analysis/Synthesis Approach to Real-Time Artificial Reverberation," Proc. IEEE ICASSP-92, San Francisco March 1992.

[6] G. Demoment, "Image Reconstruction and Restoration: Overview of Common Estimation Structures and Problems," IEEE Trans. Acoust., Speech, Signal Processing, vol. ASSP-37 (No. 12), pp. 2024-2036, Dec 1989.

[7] J. Laroche, J.M. Jot" Analysis/Synthesis of Quasi-harmonic Sounds by use of the Karplus-Strong Algorithm," these proceedings.

[8] Y. Potard, P. F. Baisnée, J. B. Barrière "Experimenting with models of resonance produced by a new technique for the analysis of impulsive sounds," Proc. of International Computer Music Conference 1986. 